\title{
Spin-flop transition driven by competing magnetoelastic anisotropy terms in a spin-spiral antiferromagnet
}

\author{
L. Benito* \\ Department of Materials Science and Metallurgy, University of Cambridge, Charles Babbage Road, Cambridge CB3 OFS, United Kingdom \\ and Departamento de Física de la Materia Condensada, Universidad de Zaragoza, 50009 Zaragoza, Spain
}

(Received 10 April 2015; revised manuscript received 5 June 2015; published 23 June 2015)

\begin{abstract}
Holmium, the archetypical system for spin-spiral antiferromagnetism, undergoes an in-plane spin-flop transition earlier attributed to competing symmetry-breaking and fully symmetric magnetoelastic anisotropy terms [Phys. Rev. Lett. 94, 227204 (2005)], which underlines the emergence of sixfold magnetoelastic constants in heavy rare earth metals, as otherwise later studies suggested. A model that encompasses magnetoelastic contributions to the in-plane sixfold magnetic anisotropy is laid out to elucidate the mechanism behind the spin-flop transition. The model, which is tested in a Ho-based superlattice, shows that the interplay between competing fully symmetric $\alpha$-magnetoelastic and symmetry-breaking $\gamma$-magnetoelastic anisotropy terms triggers the spin reorientation. This also unveils the dominant role played by the sixfold exchange magnetostriction constant, where $D_{\alpha 2}^{66} \simeq 0.32 \mathrm{GPa}$ against its crystal-field counterpart $M_{\alpha 2}^{66} \simeq-0.2 \mathrm{GPa}$, in contrast to the crystal-field origin of the symmetry-breaking magnetostriction in rare earth metals.
\end{abstract}

DOI: 10.1103/PhysRevB.91.214428

PACS number(s): 75.30.Gw, 75.50.Cc, 75.70.Cn

\section{INTRODUCTION}

The concept of spin-flop [1] transition (SFT) was restricted to field-induced spin-reorientation transitions in two-sublattice collinear antiferromagnetic systems, wherein the featured threshold-field effect that accompanies the spin jumplike transition was originally predicted by Néel [2] and later on tested in $\mathrm{CuCl}_{2} \cdot 2 \mathrm{H}_{2} \mathrm{O}$ [3]. Today, the term SFT is utilized in a broader sense, which includes a variety of field-driven spin-reorientation transitions in a wide spectrum of antiferromagnetic materials [4-9].

Heavy rare earth metals, namely Dy and Ho, are a model system for spin-spiral magnetic phases $[10,11]$, which result from the intertwined [12] interplay between an oscillating indirect exchange [13-15] and spin-orbit couplings [16,17]. A large spin-lattice [18] coupling in heavy rare earth metals gives rise to huge lattice strictions, $\sim 1 \%$, which increases the complexity of noncollinear magnetic phases, with respect to that upheld by the undistorted lattice, introducing high-order harmonics [19] into the magnetic spectrum.

High-precision vector magnetic torque experiments [20] revealed that Ho undergoes an unexpected field-induced inplane SFT, which was originally attributed to competing magnetoelastic (MEL) anisotropy [21] terms. The existence of a fully symmetric sixfold MEL coefficient $\lambda^{66}$, which gives rise to the sixfold modulation of the $\alpha$ strains $\varepsilon_{\alpha 1}$ (isotropic volume expansion) and $\varepsilon_{\alpha 2}$ (tetragonal distortion of the hexagonal cell), originates a 12-fold MEL-induced magnetic anisotropy energy (MAE) constant $K_{12}^{12}$ as experimentally confirmed in bulk holmium [22]. The existence of $\lambda^{66}$ is associated with the appearance of six-fold MEL constants, which may not be solely restricted to the Ho metal [23]. More importantly, $\lambda^{66}$, and by extent $K_{12}^{12}$, exhibits a visible nonmonotonic dependence on temperature [22]. Making use of arguments based on point-group theory [24], it is straightforward to establish that $\lambda^{66}$, as well as $K_{12}^{12}$, are encompassed by competing sixfold crystal-field (single-ion) and exchange

*luisbenito.phys@gmail.com (two-ion) MEL terms. This result is obtained from extending the Callen's theory of magnetostriction [25] to sixth order in the angular momentum operators. Additionally, the outcome of this study shows that, in contrast to the general wisdom that states that the magnetostriction (MS) in heavy rare earth metals is primarily of crystal-field origin [16,17,26,27], $\alpha$-striction modes are significantly contributed by exchange terms. Generally, two-ion MEL contributions are neglected and when these are brought into the analysis tend to be considerably smaller than single-ion counterparts [28].

In heavy rare earth metals, the Fermi surface (FS) topology is determined by the crystal lattice parameters [29], to a point where heavy $4 f$ elements are well-modeled by a crystallomagnetic phase diagram [30]. Experimentally, the ratio between the interplanar spacing $c$ and the interatomic, intraplanar spacing $a$ of the hexagonal-close-packed lattice, $c / a$, which is linked to $\varepsilon_{\alpha 2}$, seems to be the crucial parameter that triggers the electronic topological transition [31-35], supporting the nesting hypothesis [36] that links the appearance of webbing features in the FS topology to the onset of noncollinear magnetic ordering in rare earth metals.

More importantly, due to the set of spin-spiral magnetic structures in Ho, this $4 f$ metal is gathering increasing fundamental interest, because of the realization that this can be utilized as test ground for assessing new experimental techniques in condensed-matter physics [37], exploring novel concepts in hybrid superconductor/ferromagnetic spintronics [38] devices and investigating the impact that low-dimensional effects have upon the structure of noncollinear magnetic phases [39-41]. Thereby, unlocking the origin of the spin-flop transition in a model system raising wide-spread attention and finding out the influence that single- and two-ion MEL strictions possess is not only essential for a better understanding of holmium's complex magnetism, but also central for gaining a complete picture of the determining effect that magnetostrictions have upon the orientation of the spin arrangement in spin-orbit-coupling dominated rare earth metals.

This paper proposes a MEL model for the SFT in Ho, which constitutes one out of a few genuine examples of 
magnetoelastically driven spin-flop transition [8] in antiferromagnetic systems. The model confirms that $\alpha$ - and $\gamma$-MEL strictions of the hexagonal lattice originate competing in-plane MEL anisotropy terms, and their balance, jointly to the magnetocrystalline anisotropy, is the mechanism that triggers the basal-plane spin reorientation. The SFT model includes crystal-field and exchange high-order MEL constants, and is successfully tested in a Ho-based superlattice. Finally, sixfold exchange $\alpha$-MEL constants are proven larger than crystal-field counterparts in absolute value, which indicates that the anisotropic part of the volume and tetragonal MS modes are dominated by two-ion MEL interactions.

\section{THERMAL ANALYSIS OF THE VOLUME AND TETRAGONAL MAGNETOSTRICTION MODES IN HOLMIUM}

First, it is important to notice that the Callen's theory of magnetostriction [25] usually considers terms up to second order in the angular momentum operators, i.e., the magnetostrictive hexagonal solid is approximated by a crystallographic lattice with cylindrical symmetry. The differentiated aspect of this study is the starting point of our analysis, i.e., the relationships for the $\alpha \mathrm{MS}$, i.e., $\bar{\varepsilon}_{\alpha 1}$ and $\bar{\varepsilon}_{\alpha 2}$, which include coefficients up to sixth-order in the angular momentum operators (see the Appendix). The approach outlined here in order to undertake the yet not well-understood linear MS in holmium [42] consists of analyzing the temperature dependence of symmetric $\alpha$-MS modes, rather than the linear MS, which encompasses a complex mix of temperature-dependent coefficients, this approach being general to magnetic solids with hexagonal symmetry.

From the linear anomalous expansions in holmium [42] measured along the high-symmetry directions in the hexagonal lattice, $\mathbf{a}(1010), \mathbf{b}(10 \overline{1} 0)$, and $\mathbf{c}(0001)$, it is straightforward to determine the symmetric anomalous expansions [25] or spontaneous strictions $\varepsilon_{\alpha 1,2}^{s p}$, which comprise lattice (DebyeGrüneisen) and magnetoelastic (magnet-induced) lattice distortions. If the Debye-Grüneisen contribution [43] is properly taken away, the result can be attributed to the zero-field magnetostriction, $\bar{\varepsilon}_{\alpha 1,2}^{0}$. Similarly, from the linear strictions measured under a strong enough applied magnetic field [42] $\left(\mu_{0} H=3 \mathrm{~T}\right)$ that ensures the full alignment of the spin arrangement [44] along $\mathbf{H} \| b$, the symmetric field-induced (forced) strictions $\varepsilon_{\alpha 1,2}^{h}$ can be determined. Additionally, subtracting the lattice contribution [43] from $\varepsilon_{\alpha 1,2}^{h}$ yields the $\alpha$-MS modes $\bar{\varepsilon}_{\alpha 1,2}$.

Figure 1 shows the $\alpha$-MS modes in bulk Ho for $\mu_{0} H=3 \mathrm{~T}$. It is observed that $\bar{\varepsilon}_{\alpha 1}$ and $\bar{\varepsilon}_{\alpha 2}$ attain larger values at low temperature than the symmetry-breaking $\gamma$-striction in Ho. In particular, this latter attains a smaller value in Ho than in its spin-spiral counterparts, Tb and Dy (i.e., $\bar{\varepsilon}_{\gamma 1} \sim 1.3 \times 10^{-3}$ for the former against $\sim 5.7 \times 10^{-3}$ and $\sim 5.3 \times 10^{-3}$ for the latter [28], respectively). More particularly, a comparative analysis of the $\alpha$-MS modes reveals that $\bar{\varepsilon}_{\alpha 2}$ is almost $80 \%$ larger in Ho than in Dy, whereas that $\bar{\varepsilon}_{\alpha 1}$ attains a value that is about $20 \%$ bigger in Dy than in Ho (not shown here); furthermore, the ratio $\bar{\varepsilon}_{\alpha 2} / \bar{\varepsilon}_{\alpha 1}$ in Ho indicates a clear asymmetry between the $\alpha$ strictions, being considerably larger $\bar{\varepsilon}_{\alpha 2}$; however, this

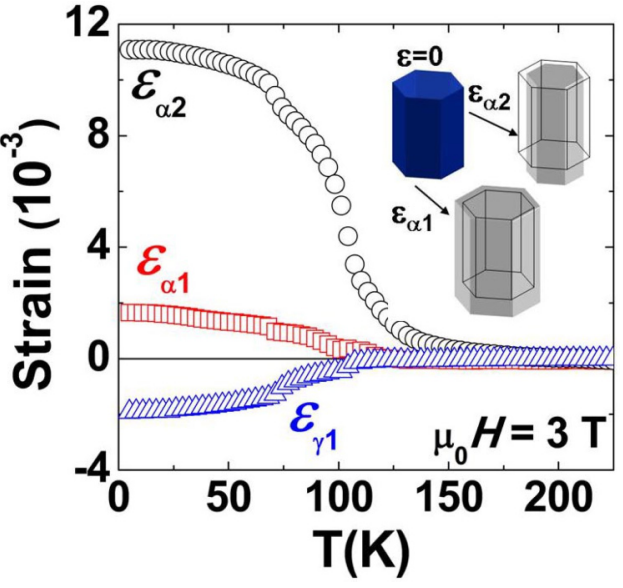

FIG. 1. (Color online) Symmetric volume $\bar{\varepsilon}_{\alpha 1}$ (black circles), tetragonal $\bar{\varepsilon}_{\alpha 2}$ (red squares), and orthorhombic $\bar{\varepsilon}_{\gamma 1}$ (blue triangles), magnetostriction (MS) modes in single-crystal Ho, belonging to the symmetric representations $\Gamma^{\alpha}$ and $\Gamma^{\gamma}$ in hexagonal symmetry [25]. $\alpha$-and $\gamma$-MS modes have been obtained working out the linear MS modes measured by Rhyne et al. [42]. For further details see text.

asymmetry is less marked in Dy where both $\alpha$-striction modes reach much alike values.

The extension of the Callen's theory of magnetostriction up to sixth order provides relationships for $\bar{\varepsilon}_{\alpha_{1,2}}$ [see the Appendix, Eq. (A17)], which can be rearranged according to its dependence on the azimuthal angle $\phi$ as follows:

$$
\bar{\varepsilon}_{\alpha_{1,2}}=\bar{\varepsilon}_{\alpha_{1,2}}^{0}+\bar{\varepsilon}_{\alpha_{1,2}}^{\phi},
$$

where $\bar{\varepsilon}_{\alpha_{1,2}}^{0}$ and $\bar{\varepsilon}_{\alpha_{1,2}}^{\phi}$ are thereafter referred to as the isotropic and anisotropic ( $\phi$-dependent) contributions to the $\alpha \mathrm{MS}$, respectively, which read as

$$
\begin{aligned}
\bar{\varepsilon}_{\alpha_{1,2}}^{0}= & \frac{1}{c_{\alpha}} \sum_{l=2,4,6}\left\{\left(M_{\alpha_{1,2}}^{l} c_{\alpha_{2,1}}-M_{\alpha_{2,1}}^{l} c_{\alpha_{3}}\right) \hat{I}_{(2 l+1) / 2}[\tilde{m}]\right. \\
& \left.+\left(D_{\alpha_{1,2}}^{l} c_{\alpha_{2,1}}-D_{\alpha_{2,1}}^{l} c_{\alpha_{3}}\right)\left[\hat{I}_{3 / 2}(\tilde{m})\right]^{2}\right\}
\end{aligned}
$$

and

$$
\begin{aligned}
\bar{\varepsilon}_{\alpha_{1,2}}^{\phi}= & \frac{1}{c_{\alpha}}\left\{\left(M_{\alpha_{1,2}}^{66} c_{\alpha_{2,1}}-M_{\alpha_{2,1}}^{66} c_{\alpha_{3}}\right) \hat{I}_{13 / 2}[\tilde{m}]\right. \\
& \left.+\left(D_{\alpha_{1,2}}^{66} c_{\alpha_{2,1}}-D_{\alpha_{2,1}}^{66} c_{\alpha_{3}}\right)\left(\hat{I}_{3 / 2}[\tilde{m}]\right)^{2}\right\} \cos 6 \phi .
\end{aligned}
$$

In zero field Ho develops a basal plane helical antiferromagnetic structure below its Néel temperature $T_{N}=132 \mathrm{~K}$ [45], where the magnetic moments within the same atomic sheet form a ferromagnetic arrangement and when moving along the $\mathbf{c}$ axis, the magnetic moments rotate a fixed angle between adjacent $c$ planes, forming a spin-spiral magnetic arrangement; below its Curie temperature $T_{\mathrm{C}}=18 \mathrm{~K}$, this structure turns into a conical spin-spiral structure, where the magnetic moments tilt away from the $c$ plane $\left(\approx 5^{\circ}\right)$ and lies in the surface of a imaginary cone, developing a small ferromagnetic component along the $\mathbf{c}$ axis.

Evaluating $\bar{\varepsilon}_{\alpha_{1,2}}$ in the helical antiferromagnetic phase that sets in Ho metal in zero field will allow us to make a crucial identification. Thus, if we sum up in Eq. (1) over all possible orientations for the magnetic moments in the $N$ ferromagnetic 

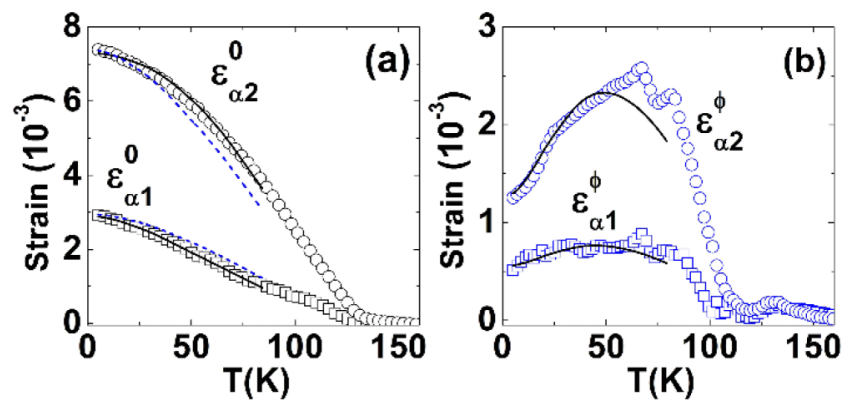

FIG. 2. (Color online) Temperature scaling of (a) the zero-field volume (squares) and tetragonal (circles) symmetric magnetostriction (MS), $\bar{\varepsilon}_{\alpha_{1}}^{0}$ and $\bar{\varepsilon}_{\alpha_{2}}^{0}$, respectively, and (b) the anisotropic sixfold volume (squares) and tetragonal (circles) MS, $\bar{\varepsilon}_{\alpha_{1}}^{\phi}$ and $\bar{\varepsilon}_{\alpha_{2}}^{\phi}$, respectively, obtained after working out the measured linear MS in bulk holmium [42], e.g., after substraction of the Debye-Grüneisen contribution [43] and determining $\bar{\varepsilon}_{\alpha_{1,2}}^{\phi}=\bar{\varepsilon}_{\alpha_{1,2}}^{h}-\bar{\varepsilon}_{\alpha_{1,2}}^{0}$, where $\bar{\varepsilon}_{\alpha_{1,2}}^{h}$ is the field-induced MS. In (a) the continuous line corresponds to a fitting function, which reads as $\bar{\varepsilon}_{\alpha_{1,2}}^{0}=\frac{1}{c_{\alpha 1} c_{\alpha 2}} \sum_{l=2,4} M_{\alpha 1,2}^{l} c_{\alpha 1,2} \hat{I}_{(2 l+1) / 2}[\hat{m}]$, where the best-fit parameters are $M_{\alpha 1}^{2}=0.09 \mathrm{GPa}, M_{\alpha 1}^{4}=0.026 \mathrm{GPa}, M_{\alpha 2}^{2}=$ $0.9 \mathrm{GPa}$, and $M_{\alpha 2}^{4}=-0.17 \mathrm{GPa}$. The dashed line corresponds to a fit, which considers single-ion terms up to $l=2$, so that the best-fit parameters are $M_{\alpha 1}^{2}=0.12 \mathrm{GPa}$ and $M_{\alpha 2}^{2}=0.74 \mathrm{GPa}$. In (b) the continuous line corresponds to a fitting function, which reads as $\bar{\varepsilon}_{\alpha_{1,2}}^{\phi}(\phi=0)=\frac{1}{c_{\alpha 1} c_{\alpha 2}}\left(M_{\alpha 1,2}^{66} c_{\alpha 2,1} \hat{I}_{13 / 2}[\tilde{m}]+D_{\alpha 1,2}^{66} c_{\alpha 2,1} m^{2}\right)$, where the best-fit parameters are $M_{\alpha 1}^{66}=-0.018 \mathrm{GPa}, M_{\alpha 2}^{66}=-0.19 \mathrm{GPa}$, $D_{\alpha 1}^{66}=0.04 \mathrm{GPa}$, and $D_{\alpha 2}^{66}=0.315 \mathrm{GPa}$. For further details see the Appendix.

sheets, i.e., over the azimuthal angle $\phi_{i}$ for each $i$ layer that conforms the spin-spiral arrangement in a bulk Ho crystal, it leads to $\sum_{i} \bar{\varepsilon}_{\alpha_{1,2}}\left(\phi_{i}\right) \equiv \bar{\varepsilon}_{\alpha_{1,2}}^{0}$, given that $\sum_{i} \cos 6 \phi_{i}=0$. Therefore, it is possible to identify the $\bar{\varepsilon}_{\alpha_{1,2}}^{0}$, given in Eq. (2), with the zero-field (isotropic) $\alpha$ MS, displayed by Fig. 2(a).

Furthermore, if we proceed with the following substraction of experimental data, namely $\varepsilon_{\alpha 1,2}^{h}-\varepsilon_{\alpha 1,2}^{s p}$, the resulting striction will be associated to the symmetric anisotropic contribution to the forced MS, $\bar{\varepsilon}_{\alpha 1,2}^{\phi}$, evaluated in this case for $\phi=0(\mathbf{H} \| b)$, which is experimentally demonstrated to be non-negligible, as displayed in Fig. 2(b). Importantly, we highlight that the existence of $\bar{\varepsilon}_{\alpha 1,2}^{\phi}$ would remain unexplained if only contributions up to $l=2$ were taken into account in Eqs. (A4) and (A7) (see the Appendix). It can be seen that $\bar{\varepsilon}_{\alpha 1,2}^{0}$ shows a monotonic temperature dependence unlike $\bar{\varepsilon}_{\alpha 1,2}^{\phi}$, which presents a nonmonotonic temperature scaling, as can be seen in Fig. 2. This experimental approach makes evident the need of including competing crystal-field and exchange sixfold MEL contributions, in order to account for $\bar{\varepsilon}_{\alpha 1,2}^{\phi}$.

The modeling of the temperature variation of the experimental $\bar{\varepsilon}_{\alpha 1,2}^{0}$ and $\bar{\varepsilon}_{\alpha 1,2}^{\phi}$ will be performed utilizing the relationships given in Eqs. (2) and (3), respectively. The criteria employed in the analysis consists of finding the best-fitting function, but using the minimum number possible of crystalfield and exchange MEL constants, and of the lowest $l$ rank possible. Besides, the MEL constants resulting from the analysis of the $\alpha$ MS must be suitable input parameters for modeling the observed SFT in Ho. Thus, aiming to elucidate the influence of single- and two-ion contributions,
TABLE I. Crystal-field (single-ion), $M_{\alpha 1,2}^{66}$ and $M_{\alpha 1,2}^{2}$, and exchange (two-ion), $D_{\alpha 1,2}^{66}$ and $D_{\alpha 1,2}^{2}, \alpha$-magnetoelastic constants associated to $\varepsilon_{\alpha 1}$ (volume striction), $\varepsilon_{\alpha 2}$ (tetragonal striction) resulting from the analysis of the temperature dependence of these latter in single-crystal Ho. The values are given in GPa.

\begin{tabular}{lllll}
\hline \hline & $M_{\alpha i}^{2}$ & $M_{\alpha i}^{4}$ & $M_{\alpha i}^{66}$ & $D_{\alpha i}^{66}$ \\
\hline$\varepsilon_{\alpha 1}$ & 0.09 & 0.026 & -0.018 & 0.04 \\
$\varepsilon_{\alpha 2}$ & 0.9 & -0.17 & -0.19 & 0.315 \\
\hline \hline
\end{tabular}

we have attempted three fitting functions for $\bar{\varepsilon}_{\alpha 1,2}^{0}$ : Fit 1 includes crystal-field MEL constants up to $l=2$; fit 2 includes crystal-field MEL constants up to $l=4$ and, finally, fit 3 includes crystal-field and exchange MEL constants up to $l=2$. The former fails to produce a fully satisfactory fit of the experimental data, apart from a narrow range at very low temperatures; fit 1 overestimates the experiment for $\bar{\varepsilon}_{\alpha 1}^{0}$ and underestimates that for $\bar{\varepsilon}_{\alpha 2}^{0}$. Fits 2 and 3 result in an almost indistinguishable fitting function (not shown here), which in both cases produces an excellent accord between experiment and theory for $\bar{\varepsilon}_{\alpha 1,2}^{0}$ [see Fig. 2(a)]. From the latter, we have opted for retaining fit 2 to the detriment of fit 3 , because the former yields a unique set of $\alpha$-MEL constants that will enable us to model the observed SFT in Ho, which is not the case of fit 3 . However, in the case of the $\bar{\varepsilon}_{\alpha 1,2}^{\phi}$, competing crystal-field and exchange MEL constants are considered, producing a relatively good agreement between the nonmonotonic temperature dependence posed by the experimental data and the model, as seen in Fig. 2(b). Figure 2 shows details for all attempted fitting functions and the best-fitting parameters calculated. We notice that the extrapolated values at $0 \mathrm{~K}$ for $c_{\alpha 1}, c_{\alpha 2}$, and $c_{\alpha 3}$ in Ho have been utilized, given that the temperature variation of the symmetric elastic constants [46] is assumed negligible in comparison to the thermal variation of the MEL constants. The values used are $c_{\alpha 1}=40 \mathrm{GPa}, c_{\alpha 2}=100 \mathrm{GPa}$, and $c_{\alpha 3}=-2 \mathrm{GPa}$.

Table I displays the best-fitting MEL constants, which result from the temperature analysis of $\bar{\varepsilon}_{\alpha 1,2}^{0}$ and $\bar{\varepsilon}_{\alpha 1,2}^{\phi}$ as explained above. At a glance, the MEL constants associated to the tetragonal MS mode $\bar{\varepsilon}_{\alpha 2}$ are around about an order of magnitude larger than those associated to the volume expansion $\bar{\varepsilon}_{\alpha 1}$. The quick analysis of the $\alpha$-MEL constants reveals the following key features: (1) The tetragonal MS mode is considerably more efficient than the volume expansion mode in decreasing the sixfold magnetic anisotropic energy, since it turns out that $M_{\alpha 2}^{66}=-0.19 \mathrm{GPa}$ and the ratio $M_{\alpha 2}^{66} / M_{\alpha 1}^{66} \simeq 10$, being fully coherent with a prior study of the impact that an in-plane compression epitaxial strain has upon the sixfold magnetic anisotropy [47]. (2) The uniaxial magnetic anisotropy increases rapidly under the appearance of $\bar{\varepsilon}_{\alpha 2}$; notice that $M_{\alpha 2}^{2}=0.9 \mathrm{GPa}$ and the ratio $M_{\alpha 2}^{2} / M_{\alpha 1}^{2}=10$. (3) $\bar{\varepsilon}_{\alpha 2}$ and $\bar{\varepsilon}_{\alpha 1}$ have dissimilar effects upon the biaxial magnetic anisotropy constant, given that $M_{\alpha 2}^{4}=-0.17 \mathrm{GPa}$ and the ratio $M_{\alpha 1}^{4} / M_{\alpha 2}^{4} \simeq-6.5$. (4) $\bar{\varepsilon}_{\alpha 2}$ notably enhances the sixfold anisotropic nature of the exchange coupling, in clear contrast to the smooth influence of $\bar{\varepsilon}_{\alpha 1}$, since the experiment shows that $D_{\alpha 2}^{66}=0.315 \mathrm{GPa}$ and the ratio $D_{\alpha 1}^{66} / D_{\alpha 2}^{66} \simeq 7.9$. Overall, the effect that the $\alpha$ strictions and, more particularly, $\bar{\varepsilon}_{\alpha 2}$ have 
upon the indirect-exchange coupling in Ho is in agreement with the findings of a recent crystallomagnetic phase diagram [30], which predicts a trend to reinforce the ferromagnetic order as the $c / a$ ratio increases.

\section{SPIN-FLOP TRANSITION MODEL IN HOLMIUM: COMPETING MEL ANISOTROPY TERMS}

The distinctive aspect of the SFT hosted by holmium is that this is observed in magnetic torque curves [21], which points to that the most likely mechanism responsible for the SFT is a field-induced competition between magnetocrystalline anisotropy, i.e., that due to the undistorted lattice, and $\alpha$ and $\gamma$-MEL anisotropy energy terms. The order parameter of the first-order spin reorientation transition is the angle $\phi$ that makes the magnetization $\mathbf{M}$ with a high-symmetry direction in the basal plane of the hexagonal-close-packed (hcp) structure. Let us commence by noticing that $M$ in Ho is confined to the basal plane by a huge uniaxial magnetic anisotropy [16] and, therefore, the relevant anisotropic part of the free energy $F_{k}$ can be written as

$$
\begin{aligned}
F_{k}= & \left\{K_{6}^{6}-\left(M_{\alpha 1}^{66}+D_{\alpha 1}^{66}\right) \varepsilon_{\alpha 1}+\left(M_{\alpha 2}^{66}+D_{\alpha 2}^{66}\right) \varepsilon_{\alpha 2}\right\} \cos 6 \phi \\
& -\sum_{l=2,4,6} M_{\gamma_{2}}^{l}\left(\varepsilon_{\gamma 1} \cos 2 \phi+\varepsilon_{\gamma 2} \sin 2 \phi\right) \\
& -\sum_{l=4,6} M_{\gamma^{4}}^{l}\left(\varepsilon_{\gamma 1} \cos 4 \phi-\varepsilon_{\gamma_{2}} \sin 4 \phi\right)
\end{aligned}
$$

where the first term corresponds to the sixfold magnetocrystalline anisotropy energy constant $K_{6}^{6}$, the next linear combination involving $\alpha$ strictions is referred to as the $\alpha$-MEL anisotropy terms, and the last two sums including $\gamma$ strictions to the $\gamma$-MEL anisotropy terms, all these terms constitute the effective sixfold MAE in the basal plane. In the case of Ho, $\phi$ is the angle that makes $M$ with the $b$ axis, i.e., holmium's easy direction for $M$ at high field [44]. It is convenient to clarify that Ho's magnetic structure turns from a spin-spiral phase in zero field into a forced ferromagnetic (FM) structure along $\mathbf{H} \| b$, passing through intermediate magnetic phases, such as for instance distorted helix and fan phases as $H$ increases [45]. Fan phases are featured by a rapid increase of the $M$, so that this latter quickly approaches to a fully magnetized state and results from an ordered bunching of the spin arrangement, posing a narrow angular dispersion around $\mathbf{H}$ [48]. Assuming that a fan phase can be visualized in first order of approximation as a pseudoferromagnetic structure, then for $H \geqslant H_{c 1}$, where $H_{c 1}$ is the critical field for the field-induced transition from a spin-spiral into a fan phase [48], the condition for $\phi=0$ to become an extreme leads to

$$
\left.\frac{\partial F_{k}}{\partial \phi}\right|_{\phi=0}=\left[M_{\gamma 2}^{2}+M_{\gamma 2}^{4}+M_{\gamma 2}^{6}+2\left(M_{\gamma 4}^{4}+M_{\gamma 4}^{6}\right)\right] \varepsilon_{\gamma 2}=0 .
$$

Now, in a general case, both $\gamma$-striction modes will be non-null and, therefore, the relationship between $\gamma$-MEL constants that must be met for high-symmetry $\mathbf{a}$ and $\mathbf{b}$ directions become extremes in a fan or forced FM phase, compatible with $\varepsilon_{\gamma 2} \neq 0$, reads as

$$
\sum_{l=2,4,6} M_{\gamma 2}^{l}=-2 \sum_{l=4,6} M_{\gamma 4}^{l}
$$

The condition that must be met for $\phi=0$ to become maximum or minimum, after inserting Eq. (6) into Eq. (5), leads to

$$
\frac{\partial^{2} F_{k}}{\partial \phi^{2}}=3\left(K_{6}^{6}-\sum_{i=1,2} M_{\alpha, i}^{66} \varepsilon_{\alpha, i}\right)+2\left(M_{\gamma 4}^{4}+M_{\gamma 4}^{6}\right) \varepsilon_{\gamma 1} .
$$

It becomes clear from inspecting Eq. (7) that the $\alpha$ - and $\gamma$ strictions, which are intrinsically functions of the temperature and the applied magnetic field, i.e., $\varepsilon_{(\Gamma) i}(T, H)$, determine the easy direction for $M$ in Ho. In other words, the balance between the different contributions to the MAE, namely the magnetocrystalline $\alpha$ - and $\gamma$-MEL anisotropy terms, is unambiguously the microscopic mechanism underlying the SFT. Thus, if $\frac{\partial^{2} F_{k}}{\partial \phi^{2}}<0$, then $\cos \phi=0$, which means that the $a$ axis is the easy direction for $M$. By contrary, if $\frac{\partial^{2} F_{k}}{\partial \phi^{2}}>0$, then $\cos \phi=1$ and the $b$ axis is the easy direction for $M$.

At this point, it is interesting to set out what high-symmetry direction in the basal plane the $\alpha$ - and $\gamma$-MEL contributions to MAE tend to align the magnetic moments. First, we should consider the sign of the symmetric MS modes at high field (as observed in Fig. 1, we notice that $\varepsilon_{\alpha 1}, \varepsilon_{\alpha 2}>0$ and $\varepsilon_{\gamma 1}<0$ ) and that of the $\alpha$-MEL (see Table I) and $\gamma$-MEL [28] constants. In addition, we must bear in mind that the symmetric MS modes in single-crystal Ho are monotonic functions of the temperature (see Fig. 1) and the applied field, such as earlier studies have shown $[18,26,27,49]$. Building on these experimental facts, we conclude that the sixfold $\alpha$-MEL and $\gamma$-MEL contributions to the MAE, which are referred to as $K_{6, m e l}^{6, \alpha}$ and $K_{6, m e l}^{6, \gamma}$ anisotropy constants, respectively, which read as

$$
\begin{aligned}
& K_{6, \text { mel }}^{6, \alpha}=-\sum_{i=1,2}\left(M_{\alpha, i}^{66}+D_{\alpha, i}^{66}\right) \varepsilon_{\alpha, i}, \\
& K_{6, \text { mel }}^{6, \gamma}=\left(M_{\gamma 2}^{4}+M_{\gamma 4}^{6}\right) \varepsilon_{\gamma 1},
\end{aligned}
$$

compete each other to align $M$ along high-symmetry directions in the basal plane of the hcp structure. Thus, if we consider that $M_{\gamma 4}^{6} \ll M_{\gamma 4}^{4} \approx-0.85 \mathrm{GPa}$ [28] (notice that experimentally the $\gamma$ MS in Ho is finely modeled by utilizing a few low-rank MEL constants [28]), which means that $K_{6, \text { mel }}^{6, \gamma}>0$, we can conclude that the $\gamma$-MEL anisotropy term tends to align $\mathbf{M}$ along the b direction, competing with $K_{6}^{6}$ [28]. On the other hand, from inspecting Eq. (8), we observe that $K_{6, \text { mel }}^{6, \alpha}<0$, which indicates that the $\alpha$-MEL anisotropy term tends to align M along the a direction, cooperating with $K_{6}^{6}$.

In order to test the proposed model for the SFT in Ho, we find it appropriate to re-arrange $K_{6, m e l}^{6, \alpha}$ and $K_{6, \text { mel }}^{6, \gamma}$. To this end, the relationships for $\bar{\varepsilon}_{\alpha 1}, \bar{\varepsilon}_{\alpha 2}$ [given in Eq. (1)] and $\bar{\varepsilon}_{\gamma 1}$ [28] will be inserted into Eq. (8), which after retaining only $\alpha$-MEL constants up to $l=2,4$, and considering that $c_{\alpha}=c_{\alpha 1} c_{\alpha 2}-c_{\alpha 3}^{2} \approx c_{\alpha 1} c_{\alpha 2}$, enables us to provide a further simplified relationship, leading to

$$
\begin{aligned}
K_{6, m e l}^{6, \alpha} \simeq & \sum_{i=1,2} \frac{-1}{c_{\alpha i}}\left(\sum_{l=2,4} M_{\alpha i}^{l} \hat{I}_{l+1 / 2}[\hat{m}]\right) \\
& \times\left(M_{\alpha i}^{66} \hat{I}_{13 / 2}[\hat{m}]+D_{\alpha i}^{66} m^{2}\right)
\end{aligned}
$$


and applying the same cutoff criteria to $\gamma$-MEL constants leads to

$$
K_{6, m e l}^{6, \gamma} \simeq \frac{-1}{c_{\gamma}}\left\{M_{\gamma 2}^{2} M_{\gamma 4}^{4} \hat{I}_{5 / 2}[\hat{m}] \hat{I}_{9 / 2}[\hat{m}]+M_{\gamma 2}^{4} M_{\gamma 4}^{4}\left(\hat{I}_{9 / 2}[\hat{m}]\right)^{2}\right\}
$$

where $c_{\gamma}=2\left(c_{11}-c_{12}\right)=106.6 \mathrm{GPa}$ [46]. Finally, the effective sixfold MAE constant can be written as

$$
K_{6}^{6, \text { eff }}=K_{6, m c}^{6} \hat{I}_{13 / 2}[\hat{m}]+K_{6, m e l}^{6, \alpha}+K_{6, m e l}^{6, \gamma} .
$$

In order to test whether the proposed MEL model, which is summarized in Eq. (11), replicates a change of sign in $K_{6}^{6, \text { eff }}$ as $H$ is swept in the $\left[\mathrm{Ho}_{85} / \mathrm{Lu}_{15}\right]_{50}$ superlattice (SL), in which the SFT was first observed [21], the first aspect we must consider is the influence that the finite size [50] of the Ho layers has upon the MEL constants. Thus, as an earlier study [47] has shown, the development of typical epitaxial strains in multilayered rare earth based systems originated a negligible alteration, if any at all, in the $\gamma$-MEL constants, however, the $\alpha$-MEL ones experienced an appreciable strain-induced modification, which is in a general case modelled as follows [23]:

$$
M_{\alpha 1,2}^{p}=\frac{M_{\alpha 1,2}^{p}(0)}{1+b^{\prime} \varepsilon_{\alpha 1,2}},
$$

where $p\left(=2,4\right.$, and 66), $M_{\alpha 1,2}^{p}(0) \equiv M_{\alpha 1,2}^{p}\left(\varepsilon_{\alpha 1,2}=0\right)$, and $b^{\prime}=10^{4}$. Let us assume that Eq. (12) is equally valid for two-ion MEL constants. Besides, we will assume that the developed epitaxial strain in the target Ho/Lu SL is mostly isotropic in the deposition plane, as a prior study suggested [47]. In this way, making the following identification $\varepsilon_{x x}=$ $\varepsilon_{x x} \equiv \varepsilon_{\|}$and $\varepsilon_{z z} \equiv \varepsilon_{\perp}$, where $\varepsilon_{\|}$and $\varepsilon_{\perp}$ are the in- and out-ofplane strains, respectively, and modeling the thickness dependence of $\varepsilon_{\|}$by the relationship $\varepsilon_{\|}=\varepsilon_{0} t_{\mathrm{Lu}} /\left(c t_{\mathrm{Lu}}+t_{\mathrm{Ho}}\right)$ [51], where $\varepsilon_{0}=\frac{a_{\mathrm{Lu}}-a_{\mathrm{Ho}}}{a_{\mathrm{Ho}}}=-0.0204$, is the lattice mismatch, $a_{\mathrm{Ho}(\mathrm{Lu})}$ is the in-plane lattice parameter [16] for $\mathrm{Ho}(\mathrm{Lu}), t_{\mathrm{Ho}, \mathrm{Lu}}$ is the $\mathrm{Ho}(\mathrm{Lu})$ nominal layer thickness $[85$ monolayers (MLs) for Ho and $15 \mathrm{MLs}$ for the Lu layers] and $c=0.95$ is a constant resulting from the ratio between $\mathrm{Ho}$ and $\mathrm{Lu}$ elastic constants and, finally, making use of the Poisson's ratio $\varepsilon_{\perp}=-2 \varepsilon_{\|} c_{13} / c_{33}$ [52], where the ratio $c_{13} / c_{33}=0.26$ [46] in bulk Ho, it is straightforward to calculate that $\varepsilon_{\alpha 1} \simeq \frac{3}{2} \varepsilon_{\|}$and $\varepsilon_{\alpha 2} \simeq-\frac{1}{4} \varepsilon_{\|}$, where $\varepsilon_{\|}=-0.00308$. For illustrative purposes, the epitaxial strain developed in the $\mathrm{Ho}_{85} / \mathrm{Lu}_{15}$ SL [in-plane compression] entails a sharp diminishing of the $\alpha$-MEL constants, which in the case of $\varepsilon_{\alpha 2}$ means that these latter solely amount to $12 \%$ out of the unstrained constant values.

Figure 3 shows the experimental SFT driven by field in the $\mathrm{Ho}_{85} / \mathrm{Lu}_{15} \mathrm{SL}$ at $T=50 \mathrm{~K}$. It is observed that the outlined model achieves an excellent agreement with the experiment. The sign crossover manifested by the field-dependent $K_{6}^{6 \text {,eff }}$ is modelled making use of Eq. (11), so that we have taking $K_{6}^{6}=-1.84 \mathrm{MPa}$ [28], $K_{6, \text { mel }}^{6, \gamma}$ as given in Eq. (10), $K_{6, \text { mel }}^{6, \alpha}$ as given in Eq. (9), the $\alpha$-MEL constants determined in this study (see Table I), and the earlier obtained $\gamma$-MEL [28] constants in Ho. Notice that we have taken into account the strain dependence of the $\alpha$-MEL applying Eq. (12) to estimate the strain-induced downsizing. The only parameter utilized is the measured $m(T=50 \mathrm{~K}, H)$ in the Ho/Lu SL, which determines

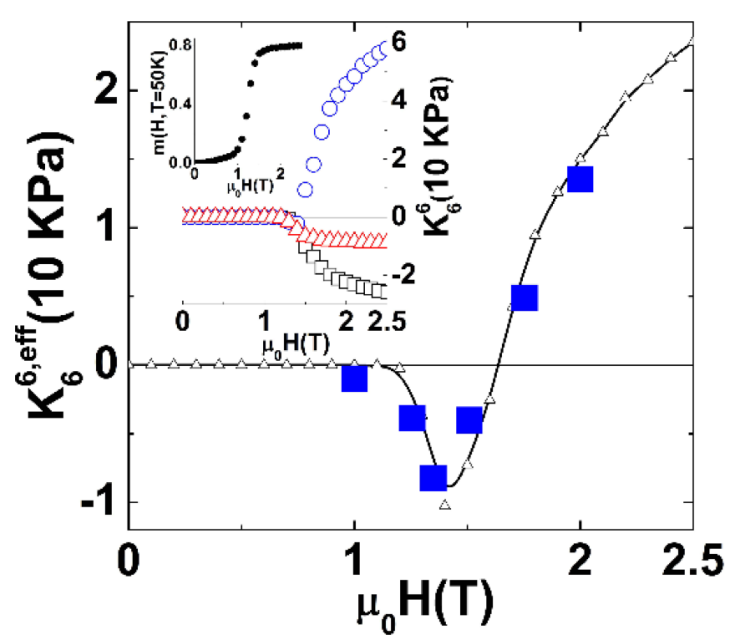

FIG. 3. (Color online) Field-dependent effective sixfold magnetic anisotropic energy (MAE) constant $K_{6}^{6, \text { eff }}$ (filled squares) experimentally determined in a $\left[\mathrm{Ho}_{85} / \mathrm{Lu}_{15}\right]_{50}[21]$ superlattice at $T=50 \mathrm{~K}$. The empty triangles correspond to the calculated values for $K_{6}^{6, \text { eff }}$ using Eq. (11) (the line serves as an eye guide). The inset graph displays the field-dependent magnetocrystalline, $\gamma$ - and $\alpha$-magnetoelastic MAE constants $K_{6, m c}^{6}$ (black squares), $K_{6, m e l}^{6, \gamma}$ (blue circles), and $K_{6, m e l}^{6, \alpha}$ (red triangles), respectively as a function of the reduced magnetization, $m(T=50 \mathrm{~K}, H)$, equally shown, utilized in the simulation of the in-plane spin-flop transition. For further details see text.

the values of the reduced hyperbolic Bessel functions. For completeness, Fig. 3 also displayes $m(T=50 \mathrm{~K}, H)$, and the calculated field-dependent magnetocrystalline $\gamma$ - and $\alpha$-MEL anisotropy terms utilized in the simulation as a function of $H$. The experimental critical field for the SFT at $T=50 \mathrm{~K}$ is $\mu_{0} H_{c}\left(K_{6}^{6, \text { eff }} \approx 0\right) \simeq 1.6 \mathrm{~T}$.

Finally, a brief remark on the discarding of fit 3 . If we proceeded in the same way as spelled out above with the crystal-field and exchange MEL constants derived from fit 3 , it is straightforward to calculate that under such a premise $K_{6, m e l}^{6, \alpha}$ would include a dominant term reading as $-1 / c_{\alpha}\left(D_{\alpha 2}^{2} D_{\alpha 2}^{66} c_{\alpha 1}+D_{\alpha 1}^{2} D_{\alpha 1}^{66} c_{\alpha 2}\right) m^{4}$, where the best-fitting parameters would be $D_{\alpha 1}^{2}=-0.1 \mathrm{GPa}, D_{\alpha 1}^{66}=0.04 \mathrm{GPa}$, $D_{\alpha 2}^{2}=0.38 \mathrm{GPa}$, and $D_{\alpha 2}^{66}=0.37 \mathrm{GPa}$. Despite taking into account the resizing effect introduced by the epitaxial strain into the declared crystal-field and exchange MEL constants [described by Eq. (12)], the aforementioned $\alpha$-MEL anisotropy term would force the spins to align along the $a$ axis, since in that situation $K_{6}^{6, \text { eff }}<0$ at any temperature and for any applied $H$.

\section{CONCLUSIONS}

As a summary, this study develops a MEL model that elucidates the mechanism behind the spin-flop transition previously observed in Ho nanostructures [21,22], which appears to be the interplay between the magnetocrystalline (undistorted lattice), $\alpha$ - and $\gamma$-MEL contributions to the in-plane sixfold magnetic anisotropy energy. Besides, the model shows that whereas $\alpha$-MEL anisotropy tends to align the spins along the $a$ axis, cooperating with the magnetocrystalline anisotropy, $\gamma$-MEL anisotropy competes with the former, tending to align 
spins along the $b$ axis. The model proposed here builds on the extension of the Callen and Callen theory of magnetostriction [25] up to sixth order in the angular momentum operators, which allows us to undertake the temperature analysis of the volume and tetragonal magnetostrictive modes in singlecrystal Ho, which has remained yet unsolved so far. From that analysis crystal-field and exchange $\alpha$-MEL constants are determined, providing essential input parameters for testing the model of the spin-flop transition in a $\left[\mathrm{Ho}_{85} / \mathrm{Lu}_{15}\right]_{50}$ superlattice. In a rare earth metal, sixfold single- and two-ion $\alpha$-MEL constants attain large values particularly in the case of the tetragonal magnetostriction mode, where $D_{\alpha_{2}}^{66}=0.365 \mathrm{GPa}$ and $M_{\alpha_{2}}^{66}=-0.2 \mathrm{GPa}$, which shows that fully symmetric magnetostriction is dominated by spin-lattice interactions with exchange origin. This investigation contributes to a better understanding of the complex magnetic phenomena arising in a model system like holmium, and by extent in heavy rare earth metals, unravelling the central role played by the MEL anisotropy energy in determining the orientation of the spins in spin-orbit-coupling dominated magnetic systems.

\section{ACKNOWLEDGMENTS}

I am deeply grateful to R. C. C. Ward for technical assistance and to M. Ciria for fruitful discussions. This work was partially supported by the $7^{\text {th }}$ Frame Program of the European Commission (MSC Actions, Contract No. 25556 MEIF-2009).

\section{APPENDIX: VOLUME AND TETRAGONAL MAGNETOSTRICTION MODES IN HEXAGONAL SYMMETRY: THE HOLMIUM CASE}

This Appendix aims to obtain the volume and tetragonal magnetostriction modes in hexagonal symmetry provided Callen's theory of magnetostriction [25] is extended to sixth order in the angular momentum operators, the maximum permitted by point-group theory. We will build on the same assumptions that Callen's theory of magnetostriction, i.e. MS is restricted to first-order effects, assuming that the spinlattice coupling is small compared to the magnetic exchange. Additionally, any dynamical coupling between the lattice and the crystal-field and magnetic exchange (crystal-field-phonon and magnon-phonon interactions) are also neglected, so that only the long-wavelength static limit is considered. The starting point is the magnetic Hamiltonian $\mathcal{H}$ for a rare earth ion located in a metallic crystal, which reads as follows: $\mathcal{H}=\mathcal{H}_{\text {exch }}+\mathcal{H}_{c f}+\mathcal{H}_{z e}$ where the Zeeman term is $\mathcal{H}_{z e}=$ $-g_{J} \mu_{B} J_{i} H$, and $g_{J}$ and $\mathbf{J}_{i}$ are the Landé factor and the total angular moment of the $i$ th ion; the indirect-exchange and crystal-field contributions per ion to $\mathcal{H}$ can be written as follows $[16,53]$ :

$$
\begin{array}{r}
\mathcal{H}_{\text {exch }}(i)=-\frac{1}{2} \sum_{(j, i)(m l)} \mathcal{K}_{l}^{m}(i, j, \bar{\varepsilon}) O_{l}^{m}(i, j), \\
\mathcal{H}_{c f}(i)=\sum_{n=2,4,6} B_{n}^{0}(i) O_{n}^{0}(i, \bar{\varepsilon})+B_{6}^{6}(i, \bar{\varepsilon}) O_{6}^{6}(i),
\end{array}
$$

where $\mathcal{K}_{l}^{m}(i, j, \bar{\varepsilon})$ and $B_{l}^{m}(i, \bar{\varepsilon})$ are the exchange and crystal-field parameters, which depend on the relative position of the rare earth ions in the solid, i.e., $\mathbf{R}_{i}-\mathbf{R}_{j}$, where $\mathbf{R}_{i}$ is the position of the $i$ th rare earth ion in the hexagonal metallic lattice, leading to the appearance of magnetoelastic interactions [54]. We have denoted the single-ion Stevens operators [55] as $O_{l}^{m}(i) \equiv O_{l}^{m}\left(\mathbf{J}_{i}\right)$ and the isomorphic two-ion ones as $O_{l}^{m}(i, j) \equiv O_{l}^{m}\left(\mathbf{J}_{i}, \mathbf{J}_{j}\right)$ for simplicity. For the sake of completeness, $\mathcal{H}_{\text {exch }}$ is introduced in the most general form, which includes anisotropic exchange contributions. The terms that appear in $\mathcal{H}_{\text {exch }}$ are found numerous and varied in nature [56], but otherwise restricted by symmetry [57]. In rare earth metals, the main and most important source of anisotropic indirect-exchange interactions [58] is the coupling between the localized and highly anisotropic $4 f$ electron clouds mediated by spin-orbit coupled $5 d-6 s$ hybridized conduction electrons [59], as a result of the large 4 forbital moment, $\mathbf{L}$, which among other effects causes the anisotropy of the magnetic moment [12]. The magnetoelastic coupling arises as a Taylor series expansion of $\mathcal{H}_{\text {exch }}$ and $\mathcal{H}_{c f}$ with respect to the components of the MS $\bar{\varepsilon}$, so that if small deformations are assumed, only linear terms in $\bar{\varepsilon}$ are considerate. It is thereby necessary to include in $\mathcal{H}$ the MEL Hamiltonian $\mathcal{H}_{\text {mel }}$, and the elastic energy per ion, $E_{e l}$. For hexagonal symmetry, $E_{e l}$ reads as [25]

$$
\begin{aligned}
E_{e l}= & \frac{1}{2} c_{\alpha 1} \varepsilon_{\alpha 1}^{2}+c_{\alpha 3} \varepsilon_{\alpha 1} \varepsilon_{\alpha 2}+\frac{1}{2} c_{\alpha 2} \varepsilon_{\alpha 2}^{2} \\
& +\frac{1}{2} c_{\gamma}\left(\varepsilon_{\gamma 1}^{2}+\varepsilon_{\gamma 2}^{2}\right)+\frac{1}{2} c_{\epsilon}\left(\varepsilon_{\epsilon 1}^{2}+\varepsilon_{\epsilon 2}^{2}\right),
\end{aligned}
$$

where the symmetric strains belonging to the symmetric representations in hexagonal symmetry are related to the Cartesian strains as follows: $\varepsilon_{\alpha 1}=\varepsilon_{x x}+\varepsilon_{y y}+\varepsilon_{z z}$, $\varepsilon_{\alpha 2}=\frac{\sqrt{3}}{2}\left(\varepsilon_{z z}-\frac{\varepsilon_{\alpha 1}}{3}\right), \quad \varepsilon_{\gamma 1}=\frac{1}{2}\left(\varepsilon_{x x}-\varepsilon_{y y}\right), \quad \varepsilon_{\gamma 2}=\varepsilon_{x y}$, $\varepsilon_{\epsilon 1}=\varepsilon_{x z}$, and $\varepsilon_{\epsilon 2}=\varepsilon_{y z}$. Equally, the symmetric elastic constants are related to the Cartesian ones by the following relationships: $c_{\alpha 1}=\frac{1}{9}\left(2 c_{11}+2 c_{12}+4 c_{13}+c_{33}\right)$, $c_{\alpha 2}=\frac{2}{3}\left(c_{11}+c_{12}-4 c_{13}+2 c_{33}\right), \quad c_{\alpha 3}=\frac{2}{3 \sqrt{3}}\left(-c_{11}-c_{12}+\right.$ $\left.c_{13}+c_{33}\right), c_{\gamma}=2\left(c_{11}-c_{12}\right)=4 c_{66}$, and $c_{\epsilon}=4 c_{44}$.

Now, considering the symmetry restrictions imposed by the point-group theory, the single- and two-ion MEL Hamiltonian per ion in hexagonal symmetry can be split into three symmetric deformation modes as follows: $\mathcal{H}_{\text {mel }}=\mathcal{H}_{\text {mel }}^{\alpha}+$ $\mathcal{H}_{\text {mel }}^{\gamma}+\mathcal{H}_{\text {mel }}^{\epsilon}$ where the single-ion MEL Hamiltonian $\mathcal{H}_{\text {mel }}^{c f}$ associated to the $\alpha$ and $\gamma$ strictions can be written as [17]

$$
\begin{aligned}
\mathcal{H}_{m e l}^{c f, \alpha}= & -\sum_{l=2,4,6}\left(\tilde{M}_{\alpha 1}^{l} \varepsilon_{\alpha 1}+\tilde{M}_{\alpha 2}^{l} \varepsilon_{\alpha 2}\right) O_{l}^{0}(i) \\
& -\left(\tilde{M}_{\alpha 1}^{66} \varepsilon_{\alpha 1}+\tilde{M}_{\alpha 2}^{66} \varepsilon_{\alpha 2}\right) O_{6}^{6}(i), \\
\mathcal{H}_{m e l}^{c f, \gamma}= & -\sum_{l=2,4,6} \tilde{M}_{\gamma 2}^{l}\left[O_{l}^{2}(i) \varepsilon_{\gamma 1}+O_{l}^{-2}(i) \varepsilon_{\gamma 2}\right] \\
& -\sum_{l=4,6} \tilde{M}_{\gamma 4}^{l}\left[O_{l}^{4}(i) \varepsilon_{\gamma 1}-O_{l}^{-4}(i) \varepsilon_{\gamma 2}\right],
\end{aligned}
$$

where $\tilde{M}_{\Gamma l}^{m}$ are the single-ion MEL parameters, which are defined as [53]

$$
\tilde{M}_{(\Gamma) l}^{m}(i)=\left.\frac{\partial B_{l}^{m}(i, \bar{\varepsilon})}{\partial \varepsilon^{\Gamma}}\right|_{\varepsilon^{\Gamma}=0} .
$$

We notice that only $\alpha$ - and $\gamma$-striction modes will be of interest for this study (Ho is an easy-plane antiferromagnet [16]), and 
thus, although $\mathcal{H}_{m e l}^{\epsilon}$ is mentioned for exactness, this will not be considered from now on. The two-ion MEL Hamiltonian $\mathcal{H}_{\text {mel }}^{\text {exch }}$, which must be invariant under time reversal and the symmetry elements of the spatial point group $D_{3 h}$, this is $\mathcal{H}_{\text {mel }}^{\text {exch }}$, must be formally isomorphous to $\mathcal{H}_{m e l}^{c f}$. Thus, for instance the $\alpha$-striction contribution to $\mathcal{H}_{m e l}^{\text {exch }}$, i.e., $\mathcal{H}_{m e l}^{\text {exch, } \alpha}$, which is of special interest to our study, reads as [24,53]

$$
\begin{aligned}
\mathcal{H}_{m e l}^{\text {exch } \alpha}= & -\sum_{l=2,4,6}\left[\widetilde{D}_{\alpha 1}^{l}(i j) \varepsilon_{\alpha 1}+\widetilde{D}_{\alpha 2}^{l}(i j) \varepsilon_{\alpha 2}\right] O_{l}^{0}(i, j) \\
& -\left[\widetilde{D}_{\alpha 1}^{66}(i j) \varepsilon_{\alpha 1}+\widetilde{D}_{\alpha 2}^{66}(i j) \varepsilon_{\alpha 2}\right] O_{6}^{6}(i, j),
\end{aligned}
$$

where in this case $\widetilde{D}_{\Gamma l}^{m}$ are the two-ion MEL parameters, which are generically defined as [53]

$$
\widetilde{D}_{(\Gamma) l}^{m}(i, j)=\left.\frac{\partial \mathcal{K}_{l}^{m}(i, j, \bar{\varepsilon})}{\partial \varepsilon^{\Gamma}}\right|_{\varepsilon^{\Gamma}=0} .
$$

The aim here is to obtain the relationships for the $\alpha$-MS modes, i.e., $\bar{\varepsilon}_{\alpha_{1,2}}$, which will enable us to undertake the temperature analysis of the experimental volume and tetragonal MS in a heavy rare earth metal. In a general case, crystal-field and exchange contributions up to sixth order in the angular momentum operators will be included in the analysis. For didactics, our starting point will be the MEL and elastic $\alpha$ Hamiltonian including only MEL interactions with crystalfield origin, which reads as

$$
\begin{aligned}
\mathcal{H}_{m e l, e l}^{\alpha}= & -\sum_{i=1}^{N} \sum_{l=2,4,6}\left(\tilde{M}_{\alpha_{1}}^{l} \varepsilon_{\alpha_{1}}+\tilde{M}_{\alpha_{2}}^{l} \varepsilon_{\alpha_{2}}\right) Q_{l}^{0}\left(J_{i}\right) \\
& -\left(\tilde{M}_{\alpha_{1}}^{66} \varepsilon_{\alpha_{1}}+\tilde{M}_{\alpha_{2}}^{66} \varepsilon_{\alpha_{2}}\right) Q_{6}^{6}\left(J_{i}\right) \\
& +N\left(\frac{1}{2} c_{\alpha 1} \epsilon_{\alpha 1}^{2}+\frac{1}{2} c_{\alpha 2} \epsilon_{\alpha 2}^{2}-c_{\alpha 3} \epsilon_{\alpha 1} \epsilon_{\alpha 1}\right),
\end{aligned}
$$

where $Q_{m}^{l}(i) \equiv Q_{m}^{l}\left(\mathbf{J}_{i}\right)$ are single-ion Stevens operators, where $\mathbf{J}_{i}$ is now the total angular momentum per ion referred to a coordinate system $(\xi, \eta, \zeta)$ parallel to the principal a, $\mathbf{b}$, and $\mathbf{c}$ directions of the hexagonal crystallographic lattice (see Fig. 4), so that $\zeta$ is the quantification axis for $\mathbf{J}$. The $\alpha$ MS are obtained by minimizing the sum of the elastic and MEL contributions to the $\alpha$-free-energy density, i.e., $F^{\alpha}=F_{e l}^{\alpha}+F_{m e l}^{\alpha}$ with respect to the $\alpha$ strains, $\varepsilon_{\alpha_{1,2}}$, so that $F_{e l}^{\alpha}$ is written classically and $F_{m e l}^{\alpha}$ is obtained by taking the thermal average of the MEL $\alpha$ Hamiltonian, i.e., $\left\langle\mathcal{H}_{\text {mel,el }}^{\alpha}\right\rangle$. In this way, resolving $\partial\left\langle\mathcal{H}^{\alpha}\right\rangle / \partial \varepsilon_{\alpha_{i}}=0$ will yield the $\alpha$ MS originated by spin-lattice interactions with crystal-field origin, $\bar{\varepsilon}_{\alpha_{1,2}}^{c f}$, which can be written as [60]

$$
\begin{aligned}
\bar{\varepsilon}_{\alpha_{1,2}}^{c f}= & \frac{N}{c_{\alpha}} \sum_{l=2,4,6}\left(\tilde{M}_{\alpha_{1,2}}^{l} c_{\alpha_{2,1}}-\tilde{M}_{\alpha_{2,1}}^{l} c_{\alpha_{3}}\right)\left\langle Q_{l}^{0}(i)\right\rangle \\
& +\left(\tilde{M}_{\alpha_{1,2}}^{66} c_{\alpha_{2,1}}-\tilde{M}_{\alpha_{2,1}}^{66} c_{\alpha_{3}}\right)\left\langle Q_{6}^{6}(i)\right\rangle .
\end{aligned}
$$

Ho develops a spin-spiral antiferromagnetic structure [45] below its Néel temperature, $T_{N}=132 \mathrm{~K}$, so that the basal plane of the hcp structure is the easy plane and the $b$ direction is the easy axis for the magnetization $M$. Due to its large sixfold magnetic anisotropy [16,28], the linear MS experiments were carried out with the applied magnetic field $H$, along the $b$ axis. Therefore, $\bar{\varepsilon}_{\alpha_{1,2}}$ must be obtained for $M$ in the basal plane of the hcp lattice. On applying a strong enough $H$ along an

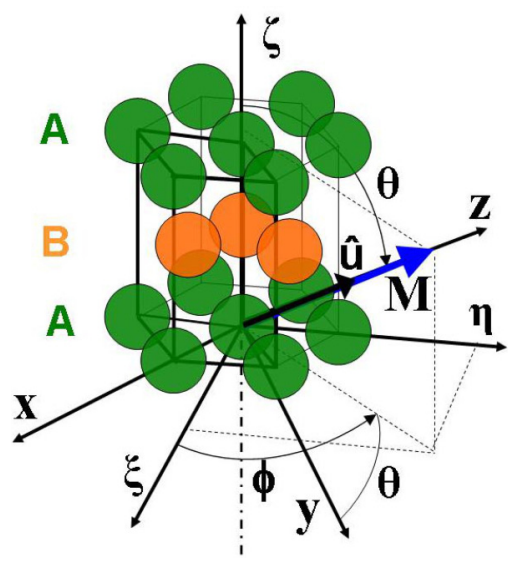

FIG. 4. (Color online) Sketch of the hexagonal-close-packed (hcp) lattice featured by heavy rare earth metals. The coordinate system $(\xi, \eta, \zeta)$ is parallel to the principal axis of the hcp lattice, i.e., $(\mathbf{a}, \mathbf{b}, \mathbf{c})$. The coordinate system $(x, y, z)$ is rotated with respect to $(\xi, \eta, \zeta)$, forming angles $(\theta, \phi)$, so that $x$ axis lies in the $(\xi, \eta)$ plane. The magnetization $\mathbf{M}$ is oriented along the direction defined by $\hat{\mathbf{u}}$.

arbitrary direction defined by the unitary vector $\hat{\mathbf{u}}$, if we choose a coordinate system $(x, y, z)$ so that it forms angles $(\theta, \phi)$ with respect to $(\xi, \eta, \zeta)$ (see Fig. 4) and $z \| \hat{\mathbf{u}}$, then assuming that $\hat{x}$ is kept in the $c$ plane of the hcp crystal lattice, it can be demonstrated that the Stevens operators $Q_{l}^{m}\left(\mathbf{J}_{i}\right)$, referred to the $(\xi, \eta, \zeta)$, can be written as a linear combination of the Stevens operators $O_{l}^{m}\left(\mathbf{J}_{i}^{\prime}\right)$ [where these latter are defined as a function of $\mathbf{J}_{i}^{\prime}$, the total angular momentum operators referred to the $(x, y, z)]$ which reads [61]

$$
Q_{l}^{m}\left(\mathbf{J}_{i}\right)=\sum_{m^{\prime}} b_{l}^{m, m^{\prime}} O_{l}^{m^{\prime}}\left(\mathbf{J}_{i}^{\prime}\right),
$$

where the coefficients of the transfer matrix are defined as $b_{l}^{m, m^{\prime}}=\left\langle O_{l}^{m^{\prime}} \mid Q_{l}^{m}\right\rangle O_{l}^{m^{\prime}}\left(\mathbf{J}_{i}^{\prime}\right)$. Given that $O_{l}^{m^{\prime}}\left(\mathbf{J}_{i}^{\prime}\right)$ equally possesses cylindrical symmetry around $\hat{\mathbf{u}}$, only those thermal averages having $m=0$ will not vanish [62]. In this way, $\left\langle Q_{l}^{m}\left(\mathbf{J}_{i}^{\prime}\right)\right\rangle_{T} \propto b_{l}^{m}\left\langle\mathrm{O}_{l}^{0}\left(\mathbf{J}_{i}^{\prime}\right)\right\rangle_{T}$, where $b_{l}^{m} \propto \mathcal{P}_{l}^{m}(\cos \theta) \cos (m \phi)$, for $m>0$ and $b_{l}^{m} \propto \mathcal{P}_{l}^{|m|}(\cos \theta) \sin (|m| \phi)$ for $m<0$, with $\mathcal{P}_{l}^{m}(\cos \theta)$ being the associated Legrendre's polynomial of the first kind. A good approximation for the thermal average $\left\langle O_{l}^{0}\left(\mathbf{J}_{i}^{\prime}\right)\right\rangle_{T}$ can be written as [63]

$$
\left\langle O_{l}^{0}(\mathbf{J})\right\rangle_{T}=c_{l} J^{(l)} \frac{I_{l+1 / 2}(\tilde{m})}{I_{1 / 2}(\tilde{m})} \equiv c_{l} J^{(l)} \hat{I}_{l+1 / 2}(\tilde{m}),
$$

where $\hat{I}_{l+1 / 2}(\tilde{m})$ is the reduced hyperbolic Bessel function and $\tilde{m} \equiv \mathcal{L}^{-1}[m(T)]$ is the inverse of the Langevin's function, $m$ is the reduced magnetization, $c_{l}$ are numerical constants, and $J^{(l)} \equiv J(J-1)\left(J-\frac{1}{2}\right) \ldots\left(J-\frac{(l-1)}{2}\right)$. Now, if one considers that $\mathbf{M}$ lies in the basal plane, the rotation of the angular momentum operators described by Eq. (A11) should be evaluated for $\theta=\pi / 2$ [61]. Doing so, inserting this result in Eq. (A10) and evaluating the thermal averages accordingly to Eq. (A12), we then obtain that the crystal-field $\alpha \mathrm{MS}$, which reads as

$$
\begin{aligned}
\bar{\varepsilon}_{\alpha_{1,2}}^{c f}= & \frac{1}{c_{\alpha}} \sum_{l=2,4,6}\left(M_{\alpha_{1,2}}^{l} c_{\alpha_{2,1}}-M_{\alpha_{2,1}}^{l} c_{\alpha_{3}}\right) \hat{I}_{l+1 / 2}[\tilde{m}] \\
& +\left(M_{\alpha_{1,2}}^{66} c_{\alpha_{2,1}}-M_{\alpha_{2,1}}^{66} c_{\alpha_{3}}\right) \hat{I}_{l+1 / 2}[\tilde{m}] \cos (6 \phi),
\end{aligned}
$$


where $M_{\alpha_{1,2}}^{l}$ and $M_{\alpha_{2,1}}^{66}$ are MEL constants related to the MEL parameters as follows: $M_{\alpha_{1,2}}^{l} \propto J^{(l)} \tilde{M}_{\alpha_{1,2}}^{l} \alpha_{l}\left\langle r_{4 f}^{l}\right\rangle$ and $M_{\alpha_{1,2}}^{66} \propto$ $J^{(6)} \tilde{M}_{\alpha_{1,2}}^{66} \beta_{J}\left\langle r_{4 f}^{6}\right\rangle$, where $\alpha_{l}\left(=\left\langle J\left\|O_{l}\right\| J\right\rangle\right)$ is the $l$ th Stevens factor [64], this is $\alpha_{l} \equiv \alpha_{J}, \gamma_{J}$, and $\beta_{J}$, for $l=2,4$, and 6 , respectively, and $\left\langle\mathrm{r}_{4 f}^{l}\right\rangle$ is the $l$ th multipole [65] of the $4 f$ wave functions [66].

At this point, it is now appropriate to obtain the $\alpha$ MS due to spin-lattice interactions with exchange origin, $\bar{\varepsilon}_{\alpha_{1,2}}^{e x c h}$. If we proceed in the same way as done in the case of the single-ion contributions to $\mathcal{H}_{m e l, e l}^{\alpha}$, bearing in mind the existing isomorphism between $\mathcal{H}_{\text {mel }}^{c f, \alpha}$ and $\mathcal{H}_{\text {mel }}^{\text {exch } \alpha}$, which is clear from inspecting Eqs. (A4) and (A8), we could conclude that $\bar{\varepsilon}_{\alpha_{1,2}}^{\text {exch }}$ can be written as [24]

$$
\begin{aligned}
\bar{\varepsilon}_{\alpha_{1,2}}^{\text {exch }}=\frac{N}{c_{\alpha}} & \sum_{l=2,4,6}\left(\widetilde{D}_{\alpha_{1,2}}^{l} c_{\alpha_{2,1}}-\widetilde{D}_{\alpha_{2,1}}^{l} c_{\alpha_{3}}\right)\left\langle Q_{l}^{0}(i, j)\right\rangle \\
& +\left(\widetilde{D}_{\alpha_{1,2}}^{66} c_{\alpha_{2,1}}-\widetilde{D}_{\alpha_{2,1}}^{66} c_{\alpha_{3}}\right)\left\langle Q_{6}^{6}(i, j)\right\rangle
\end{aligned}
$$

and, thereby, $\bar{\varepsilon}_{\alpha_{1,2}}^{\text {exch }}$ is isomorphic to $\bar{\varepsilon}_{\alpha_{1,2}}^{c f}$, given by Eq. (A11). As done in the case of the $\alpha$ MS with crystal-field origin, $Q_{m}^{l}\left(\mathbf{J}_{i}, \mathbf{J}_{j}\right)$ must be obtained for the case of $\mathbf{M} \perp \mathbf{c}$. We notice that under a rotation of coordinate system, see Fig. 4, $Q_{m}^{l}\left(\mathbf{J}_{i}, \mathbf{J}_{j}\right)$ transforms isomorphically as its equivalent classical spherical harmonic does. In such a situation, only rotations of the two-ion angular momentum complex as a whole are of interest [67], that is the two-ion total angular momentum complex acts like a single ion of angular momentum $2 \mathbf{J}$. Thus, the relation between $Q_{m}^{l}\left(\mathbf{J}_{i}, \mathbf{J}_{j}\right)$ and $O_{m}^{l}\left(\mathbf{J}_{i}^{\prime}, \mathbf{J}_{j}^{\prime}\right)$ reads as $Q_{l}^{m}(i, j)=\sum_{m^{\prime}} b_{l}^{m, m^{\prime}} O_{l}^{m^{\prime}}(i, j)$ where the coefficients $b_{l}^{m, m^{\prime}}$ are the same as those resulting from the rotation of single-ion
Stevens operators [see Eq. (A12)]. Equally, the two-ion angular momentum complex possesses cylindrical symmetry around the quantization axis, i.e., $\hat{u} \| \mathbf{M}$ in the case of the new $(x, y, z)$ and, therefore, the thermal average of $Q_{l}^{m}\left(\mathbf{J}_{i}, \mathbf{J}_{j}\right)$ can be obtained as well: $\left\langle Q_{l}^{m}\left(\mathbf{J}_{i}^{\prime}, \mathbf{J}^{\prime}{ }_{j}\right)\right\rangle \propto b_{l}^{m}\left\langle O_{l}^{0}\left(\mathbf{J}^{\prime}{ }_{i}, \mathbf{J}^{\prime}{ }_{j}\right)\right\rangle$. One requires to turn to the ubiquitous Hartree-Fock decoupling approximation [25] to evaluate the thermal average of two-ion Stevens operators [12,28], which leads to

$$
\left\langle O_{6}^{0}\left(\mathbf{J}^{\prime}{ }_{i}, \mathbf{J}^{\prime}{ }_{j}\right)\right\rangle \equiv\left\langle O_{2}^{0}\left(\mathbf{J}_{i}^{\prime}, \mathbf{J}_{j}^{\prime}\right)\right\rangle=\left\langle J_{i \zeta} J_{j \zeta}\right\rangle \propto m^{2} .
$$

Evaluating the rotation of $Q_{l}^{m}(i, j)$ for the case in which $\mathbf{M}$ is in the basal plane, i.e., for $\theta=\pi / 2$, and inserting $\left\langle O_{6}^{0}\left(\mathbf{J}_{i}^{\prime}, \mathbf{J}^{\prime}{ }_{j}\right)\right\rangle$ given by Eq. (A15) into Eq. (A14), the $\alpha$ MS with exchange origin can be written as follows:

$$
\begin{aligned}
\bar{\varepsilon}_{\alpha_{1,2}}^{\text {exch }}= & \frac{1}{c_{\alpha}}\left\{\sum_{l=2,4,6}\left(D_{\alpha_{1,2}}^{l} c_{\alpha_{2,1}}-D_{\alpha_{2,1}}^{l} c_{\alpha_{3}}\right)\right. \\
& \left.+\left(D_{\alpha_{1,2}}^{66} c_{\alpha_{2,1}}-D_{\alpha_{2,1}}^{66} c_{\alpha_{3}}\right) \cos (6 \phi)\right\} m^{2},
\end{aligned}
$$

where $D_{\alpha_{1,2}}^{l}$ and $D_{\alpha_{1,2}}^{66}$ are the two-ion MEL constants, which are related to the exchange MEL parameters through relationships which are isomorphic to the case of single-ion MEL constants. The total $\alpha$ MS is obtained by adding up the $\alpha$ MS due to spin-lattice interactions with crystal-field and exchange origin and given in Eqs. (A13) and (A16), respectively. Doing so leads to

$$
\bar{\varepsilon}_{\alpha_{1,2}}=\bar{\varepsilon}_{\alpha_{1,2}}^{c f}+\bar{\varepsilon}_{\alpha_{1,2}}^{\text {exch }} .
$$

[1] J. Ubbink, J. A. Poulis, H. J. Gerritsen, and C. J. Gorter, Physica 19, 928 (1953).

[2] L. Néel, Ann. Phys. (Paris) 5, 232 (1936); Proc. Phys. Soc. (London) A 65, 869 (1952).

[3] N. J. Poulis, J. van den Handel, J. Ubbink, J. A. Poulis, and C. J. Gorter, Phys. Rev. 82, 552 (1951).

[4] Y. Koike et al., Phys. Rev. Lett. 89, 077202 (2002).

[5] T. Fries, Y. Shapira, F. Palacio, M. C. Morón, G. J. McIntyre, R. Kershaw, A. Wold, and E. J. McNiff Jr., Phys. Rev. B 56, 5424 (1997).

[6] W. Wernsdorfer, N. Aliaga-Alcalde, D. N. Hendrickson, and G. Christou, Nature (London) 416, 406 (2002).

[7] I. Tsukada, J. Takeya, T. Masuda, and K. Uchinokura, Phys. Rev. Lett. 87, 127203 (2001).

[8] M. D. Lumsden, B. C. Sales, D. Mandrus, S. E. Nagler, and J. R. Thompson, Phys. Rev. Lett. 86, 159 (2001).

[9] R. W. Wang, D. L. Mills, E. E. Fullerton, J. E. Mattson, and S. D. Bader, Phys. Rev. Lett. 72, 920 (1994).

[10] T. Chattopadhyay, Science 264, 226 (1994).

[11] F. Li, T. Nattermann, and V. L. Pokrovsky, Phys. Rev. Lett. 108, 107203 (2012).

[12] L. Benito, K. Dumesnil, and R. C. C. Ward, Phys. Rev. B 90, 054407 (2014).

[13] H. B. Møller and J. C. G. Houmann, Phys. Rev. Lett. 16, 737 (1966).
[14] H. B. Møller, J. C. G. Houmann, and A. R. Mackintosh, Phys. Rev. Lett. 19, 312 (1967).

[15] R. M. Nicklow, N. Wakabayashi, M. K. Wilkinson, and R. E. Reed, Phys. Rev. Lett. 26, 140 (1971).

[16] Magnetic Properties of Rare Earth Metals, edited by R. J. Elliot (Plenum, London, 1972).

[17] J. Jensen and A. R. Mackintosh, Rare Earth Magnetism (Clarendon, Oxford, 1991).

[18] S. Legvold, J. Alstad, and J. Rhyne, Phys. Rev. Lett. 10, 509 (1963).

[19] Doon Gibbs, D. E. Moncton, K. L. D'Amico, J. Bohr, and B. H. Grier, Phys. Rev. Lett. 55, 234 (1985).

[20] L. Benito, J. I. Arnaudas, and A. del Moral, Rev. Sci. Instrum. 77, 025101 (2006).

[21] L. Benito, M. Ciria, C. de la Fuente, J. I. Arnaudas, R. C. C. Ward, and M. R. Wells, Phys. Rev. Lett. 94, 227204 (2005).

[22] L. Benito, M. Ciria, A. Fraile, D. Fort, J. S. Abell, and J. I. Arnaudas, Phys. Rev. Lett. 98, 267201 (2007).

[23] L. Benito, C. Ballesteros, and R. C. C. Ward, Phys. Rev. B 89, 134421 (2014).

[24] L. Benito, Ph.D. thesis, Universidad de Zaragoza, 2004.

[25] E. R. Callen and H. B. Callen, Phys. Rev. 129, 578 (1963); 139, A455 (1965). 
[26] M. Ciria, J. I. Arnaudas, A. del Moral, G. J. Tomka, C. de la Fuente, P. A. J. de Groot, M. R. Wells, and R. C. C. Ward, Phys. Rev. Lett. 75, 1634 (1995).

[27] M. Ciria, J. I. Arnaudas, A. del Moral, M. R. Wells, and R. C. C. Ward, Appl. Phys. Lett. 72, 2044 (1998).

[28] L. Benito, J. I. Arnaudas, M. Ciria, C. de la Fuente, and A. del Moral, J. Phys.: Condens. Matter 16, 7151 (2004).

[29] A. P. Cracknell and K. C. Wong, The Fermi Surface (Clarendon, Oxford, 1973).

[30] I. D. Hughes, M. Däne, A. Ernst, W. Hergert, M. Lüders, J. Poulter, J. B. Staunton, A. Svane, Z. Szotek, and W. M. Temmerman, Nature 446, 650 (2007).

[31] A. Vl. Andrianov, J. Magn. Magn. Mater. 140-144, 749 (1995).

[32] A. Vl. Andrianov and O. D. Chistiakov, Phys. Rev. B 55, 14107 (1997).

[33] A. Vl. Andrianov, D. I. Kosarev, and A. I. Beskrovnyi, Phys. Rev. B 62, 13844 (2000).

[34] A. V1. Andrianov and O. A. Savel'eva, Phys. Rev. B 67, 012405 (2003).

[35] A. Vl. Andrianov, O. A. Savel'eva, E. Bauer, and J. B. Staunton, Phys. Rev. B 84, 132401 (2011).

[36] H. M. Fretwell, S. B. Dugdale, M. A. Alam, D. C. R. Hedley, A. Rodriguez-Gonzalez, and S. B. Palmer, Phys. Rev. Lett. 82, 3867 (1999).

[37] Doon Gibbs, D. R. Harshman, E. D. Isaacs, D. B. McWhan, D. Mills, and C. Vettier, Phys. Rev. Lett. 61, 1241 (1988).

[38] J. W. A. Robinson, J. D. S. Witt, and M. G. Blamire, Science 329, 59 (2010).

[39] E. Weschke, H. Ott, E. Schierle, C. Schüßler-Langeheine, D. V. Vyalikh, G. Kaindl, V. Leiner, M. Ay, T. Schmitte, H. Zabel, and P. J. Jensen, Phys. Rev. Lett. 93, 157204 (2004).

[40] F. Cinti, A. Cuccoli, and A. Rettori, Phys. Rev. B 78, 020402(R) (2008).

[41] S. Konings, C. Schüßler-Langeheine, H. Ott, E. Weschke, E. Schierle, H. Zabel, and J. B. Goedkoop, Phys. Rev. Lett. 106, 077402 (2011).

[42] J. J. Rhyne, S. Legvold, and E. T. Rodine, Phys. Rev. 154, 266 (1967).

[43] N. W. Ashcroft and N. D. Mermin, Solid State Physics (Holt, New York, 1976).

[44] D. L. Strandburg, S. Legvold, and F. H. Spedding, Phys. Rev. 127, 2046 (1962).
[45] W. C. Koehler, J. W. Cable, M. K. Wilkinson, and E. O. Wollan, Phys. Rev. 151, 414 (1966); W. C. Koehler, J. W. Cable, H. R. Child, M. K. Wilkinson, and E. O. Wollan, ibid. 158, 450 (1967).

[46] S. B. Palmer, J. Phys. Chem. Solids 31, 143 (1970).

[47] L. Benito, J. I. Arnaudas, M. Ciria, C. de la Fuente, A. del Moral, R. C. C. Ward, and M. R. Wells, Phys. Rev. B 70, 052403 (2004).

[48] L. Benito, R. C. C. Ward, and M. G. Blamire, Phys. Rev. B 88, 224407 (2013).

[49] H. Ohsumi, K. Tajima, N. Wakabayashi, Y. Shinoda, K. Kamishima, and T. Goto, J. Phys. Soc. Jpn. 66, 1896 (1997).

[50] S. W. Sun and R. C. O'Handley, Phys. Rev. Lett. 66, 2798 (1991); R. C. O’Handley and S. W. Sun, J. Magn. Magn. Mater. 104-107, 1717 (1992).

[51] J. I. Arnaudas et al., in Frontiers in Magnetism of Reduced Dimensions Systems, edited by V. G. Baryakhtar, P. E. Wigen, and N. A. Lesnik (Kluwer Academic, Dordrecht, 1998), p. 525.

[52] D. Sander, J. Phys.: Condens. Matter 16, R603 (2004).

[53] M. Doerr, M. Rotter, and A. Lindbaum, Adv. Phys. 54, 1 (2005).

[54] E. du Tremolet de Lacheisserie, Magnetostriction: Theory and Applications of Magnetoelasticity (CRC, Boca Raton, 1993).

[55] M. T. Hutchings, Solid State Physics (Academic, New York, 1964), Vol. 16, p. 227.

[56] W. P. Wolf, J. Phys. Colloques 32, C1-26 (1971).

[57] J. Jensen, Physica B 86-88, 32 (1977).

[58] J. Jensen, J. G. Houmann, and H. B. Møller, Phys. Rev. B 12, 303 (1975)

[59] P. M. Levy, Solid State Commun. 7, 1813 (1969).

[60] O. Danielsen, Quantum Mechanical Operator Equivalents and Magnetic Anisotropy of Heavy Rare Earth Metals, Risø Report No. 295, Danish Atomic Energy Commission, 1973.

[61] O. Danielsen and P.-A. Lindgård, Quantum Mechanical Operator Equivalents Used in the Theory of Magnetism, Ris $\varnothing$ Report No. 259, Danish Atomic Energy Commission, 1972.

[62] J. H. van Vleck, Phys. Rev. 52, 1178 (1937).

[63] E. R. Callen and H. B. Callen, J. Phys. Chem. Solids 27, 1271 (1966).

[64] K. W. H. Stevens, Proc. Phys. Soc. (London) A65, 209 (1952).

[65] T. Kasuya, in Magnetism, edited by G. T. Rado and H. Suhl (Academic, New York, 1966), Vol. IIB.

[66] R. E. Watson and A. J. Freeman, Phys. Rev. 131, 250 (1963); 132, 706 (1963).

[67] J. H. Van Vleck, J. Phys. Radium 20, 124 (1959). 